\title{
Assessment of anti-CarP antibodies, disease activity and quality of life in rheumatoid arthritis patients on conventional and biological disease-modifying antirheumatic drugs
}

\author{
Sudhir Kumar ${ }^{1}$, Ghanshyam Pangtey ${ }^{1}$, Rachna Gupta ${ }^{2}$, Harmeet Singh Rehan ${ }^{1}$, Lalit Kumar Gupta ${ }^{1}$ \\ ${ }^{1}$ Lady Hardinge Medical College \& Smt. S.K. Hospital, New Delhi, India \\ 2University College of Medical Sciences, New Delhi, India
}

\begin{abstract}
Objective: Good biomarkers are important to guide decisions in the clinical management of rheumatoid arthritis (RA). RA patients harbor antibodies directed against carbamylated proteins which may predict joint damage. This study investigated whether antibodies against carbamylated proteins (anti-CarP) may serve as surrogate prognostic markers.

Material and methods: Fifty-three patients with a diagnosis of rheumatoid arthritis according to ACR 1987 criteria were included. Blood samples were analyzed for CarP antibody levels using the ELISA method. Quality of life (QoL) was assessed by the WHO SF-36 questionnaire, and disease activity was assessed using the DAS28 calculator. Newly diagnosed patients were assessed at the first visit and at 12 weeks of treatment, while a single assessment was made for patients already on maintenance therapy.

Results: Out of 53 patients, 22 had titers of anti-CarP above the cut-off range and considered as positive for anti-CarP antibodies. Anti-CarP antibody serum level was significantly higher in patients with deformity of joints and with erosions in comparison to those without any destructive changes $(p<0.05)$. There was a weak positive correlation between anti-CarP and DAS $28(p>0.05)$. Also there was a weak negative correlation in all domains of quality of life with anti-CarP antibody titers ( $p>0.05$ ). There was no significant correlation between titers of anti-CarP antibodies and presence or absence of rheumatoid factor.

Conclusions: Serum levels of anti-CarP antibodies in RA patients with joint erosions/deformities were much higher than in those without any joint damage. Anti-CarP antibodies may have good prognostic value in RA patients with erosions. Disease activity and QoL of RA patients improved during treatment, but no correlation was found between DAS 28/QoL and anti-CarP antibody serum levels.
\end{abstract}

Key words: rheumatoid arthritis, anti-CarP (anti-carbamylated protein antibodies), quality of life.

\section{Introduction}

Rheumatoid arthritis (RA) is a chronic inflammatory disease of not completely determined etiology. It is characterized by symmetric, peripheral polyarthritis with a variety of extra-articular manifestations [1]. The prevalence varies between $0.3 \%$ and $1 \%$ in developed countries [2]. Different cytokine patterns in the synovium of patients with early arthritis [3] support the hypothesis that early arthritis is characterized by different pathophysiology than in later stages. In clinical practice, a substantial majority of rheumatologists now adhere to a definition of early RA which limits the duration of the disease up to 6 months [4]. 
Autoantibodies with the highest specificity for RA are directed against proteins containing citrulline epitopes. These antibodies were first described as antiperinuclear factor antibodies (APF) [5]. High serum levels of autoantibodies such as rheumatoid factor (RF) and anti-citrullinated peptide antibodies (ACPA) can be present for up to 5 years before the clinical onset of arthritis [6]. A novel assay that specifically detects the presence of antibodies directed against carbamylated proteins (anti-CarP) [7] shows that RA patients also harbor these antibodies which may predict joint damage in the course of this disease. Anti-carbamylated protein antibodies recognize proteins only after the enzymatic conversion of the amino acid arginine by peptidyl-arginine deiminase (PAD) enzymes to become the amino acid citrulline [8, 9].

Despite conventional treatment, RA still has many deleterious consequences. From the patients' perspective, these include persistent pain, functional disability, fatigue, and depression modified by health beliefs and underlying psychological problems. Considering that only a few studies have investigated the effect of disease-modifying antirheumatic drugs (DMARDs) on anti-CarP antibodies and data correlating the effect of therapy on surrogate prognostic markers and the quality of life scores in RA patients is also lacking, we planned this study.

\section{Material and methods}

The study was conducted after clearance from the institutional Ethical Committee for Human Research and patients gave written informed consent for study. It was an open label, observational, prospective clinical study. Fifty-three RA patients who attended the medicine outpatient rheumatic clinic from December 2013 to April 2015 taking DMARDs and ten age- and gender-matched healthy volunteers were included. Diagnosis of RA was based on American College of Rheumatology 1987 Criteria. Patients with RA associated with another connective tissue disease (systemic lupus erythematosus, scleroderma, etc.), pregnant women, lactating mothers, and non-consenting patients were excluded. Clinicians performed physical and radiological examination of the patients to confirm or exclude joint damage. Then they were classified as patients with or without joint erosions/deformities. Blood samples were collected and the following parameters were estimated in all the patients: serum anti-CarP antibody concentration, complete blood count, erythrocyte sedimentation rate (ESR) and rheumatoid factor (RF). Serum samples were batch analyzed for CarP antibodies levels using enzyme-linked immunosorbent assay (ELISA) (Bmassay, Beijing, China). Quality of life was assessed by the WHO SF-36 [10] questionnaire. Disease activity was assessed by DAS 28 [11]. Figure 1 shows a summary of recruitment

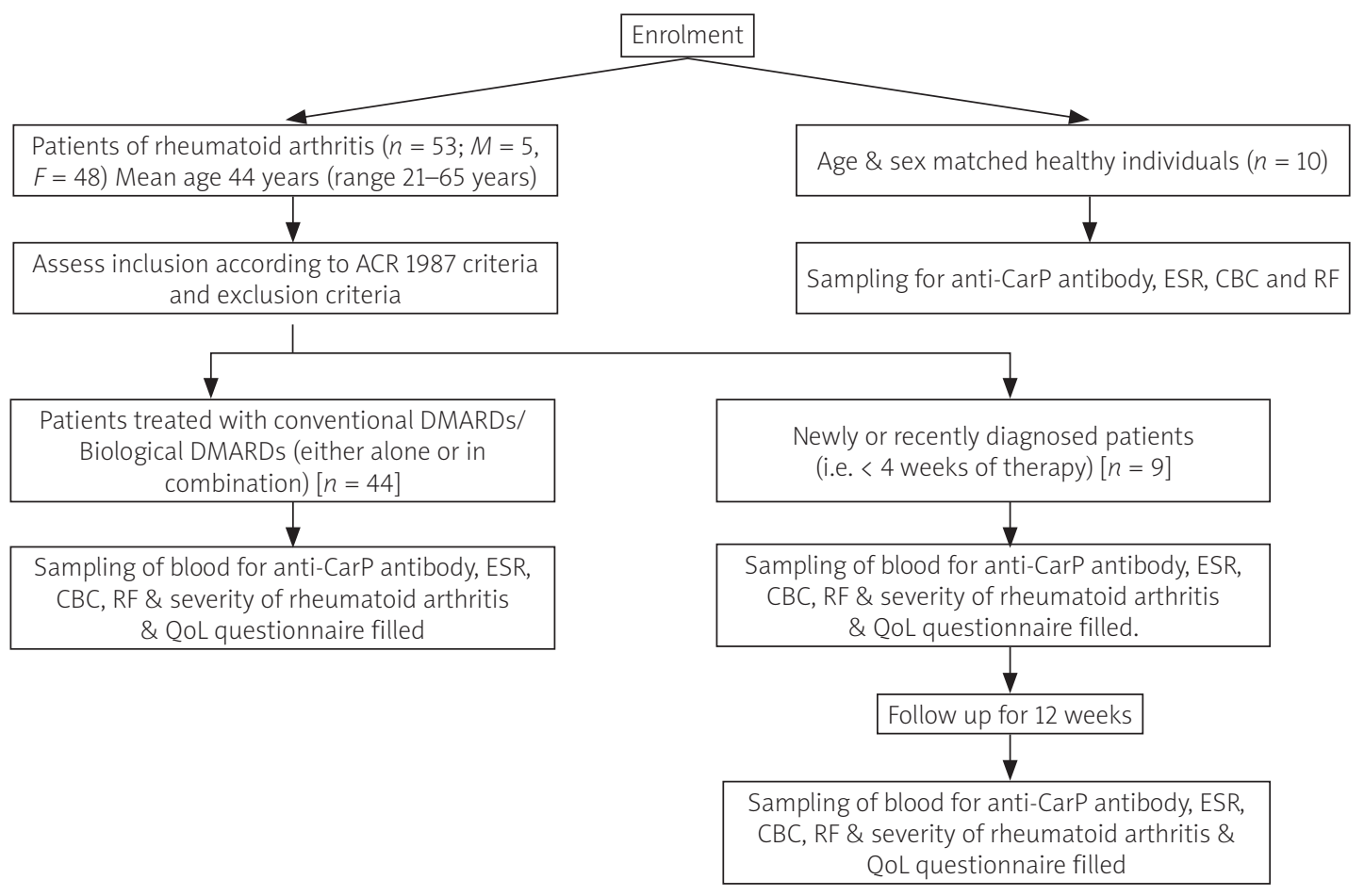

Fig. 1. Flow diagram showing patient recruitment, demographics and study methodology. 
Table I. Demographic parameters and base line characteristics of participants

\begin{tabular}{|lcc|}
\hline & Patients & Controls \\
\hline $\begin{array}{l}\text { Age (mean } \pm \text { SD) } \\
\text { (years })\end{array}$ & $43.96 \pm 10.56$ & $45.3 \pm 9.91$ \\
\hline $\begin{array}{l}\text { Gender } \\
\text { (male/female) }\end{array}$ & $5 / 48$ & $1 / 9$ \\
\hline $\begin{array}{l}\text { BP (systolic) } \\
\text { (mm Hg) }\end{array}$ & $122.79 \pm 3.87$ & $121.8 \pm 4.14$ \\
\hline $\begin{array}{l}\text { BP }(\text { diastolic) } \\
\text { (mm Hg) }\end{array}$ & $80.45 \pm 2.76$ & $81.2 \pm 2.04$ \\
\hline Haemoglobin $(\mathrm{g} \%)$ & $11.52 \pm 1.41$ & $11.93 \pm 0.85$ \\
\hline TLC $(/ \mu l)$ & $7384.34 \pm 2564.38$ & $6040.0 \pm 1276.09$ \\
\hline ESR $(\mathrm{mm} / \mathrm{hr})$ & $37.32 \pm 19.55$ & $9.2 \pm 1.94$ \\
\hline RBC $\left({ }^{*} 10^{6} / \mu l\right)$ & $4.11 \pm 0.56$ & $3.89 \pm 0.45$ \\
\hline Anti-CarP $(\mathrm{U} / \mathrm{ml})$ & $305.30 \pm 349.47$ & $148.03 \pm 3.87$ \\
\hline
\end{tabular}

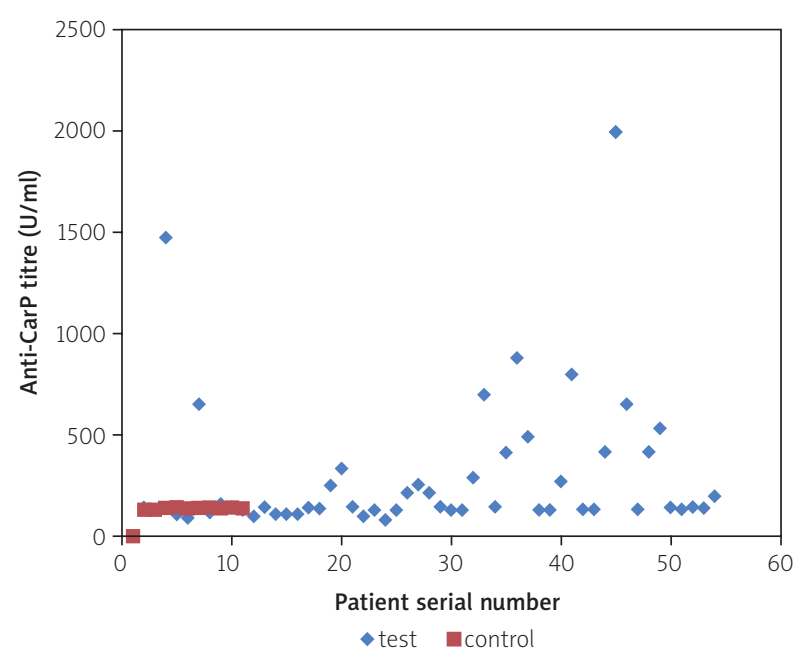

Fig. 2. Anti-CarP antibody titres in patients of rheumatoid arthritis and in matched controls.

and demographic profile and methodology used in the study subjects.

\section{Steps for estimation of serum anti-CarP antibodies}

The human serum anti-CarP polyclonal antibodies were pre-coated onto 96-well plates, then the following instructions were followed. Add $100 \mu \mathrm{l}$ of prepared serum samples or standards and incubate the plate at $37^{\circ} \mathrm{C}$ for $90 \mathrm{~min}$, then wash the plate twice with $300 \mu \mathrm{l}$ of Tris-buffered saline (TBS). Add $100 \mu$ biotinylated antibodies and incubate the plate at $37^{\circ} \mathrm{C}$ for $60 \mathrm{~min}$, then wash the plate 3 times with TBS. Add $100 \mu \mathrm{l}$ of avidin-biotin-peroxidase complex working solution and incubate the plate at $37^{\circ} \mathrm{C}$ for $30 \mathrm{~min}$, then wash the plate 5 times with TBS. Add $90 \mu$ l of 3,3',5,5'-tetramethylbenzidine (TMB) color developing agent and incubate at $37^{\circ} \mathrm{C}$. Add $100 \mu \mathrm{l}$ of stop solution. Optical density (OD) absorbance at $450 \mathrm{~nm}$ is read within $30 \mathrm{~min}$. The intensity of the color is proportional to the amount of human anti-CarP antibodies bound in samples.

The primary outcome of this study was to assess the serum level of anti-CarP antibodies of RA patients treated with DMARDs. The secondary outcome was to demonstrate the correlation of anti-CarP antibodies with the disease activity and quality of life score in RA patients after treatment with DMARDs.

Use of concomitant medications for treating related diseases such as hypertension, dyslipidaemia, diabetes, etc., was not adjusted. Any biochemical analytical bias was adjusted by keeping the analyst blind. The laboratory personnel had no knowledge of the case or control status of the samples.

The statistical analysis was performed using Microsoft Excel and SPSS, version 12. Analysis of any significant change in the serum values of the marker were compared using Student's t-test. A correlation study between anti-CarP antibody and antirheumatic drugs and quality of life was performed using Pearson's coefficient of correlation ( $r$ value). $P<0.05$ was considered statistically significant.

\section{Results}

Table I shows baseline demographic and biochemical parameters of control group and RA patients.

Out of 53 patients, 22 were found to have a concentration of anti-CarP above the cut-off range, considered positive for anti-CarP antibodies. The cut-off for positivity was calculated by the mean plus 2 standards deviation (mean +2 SD) of the titer of anti-carbamylated protein antibodies in healthy subjects. It was calculated as $155.77 \mathrm{U} / \mathrm{ml}$. Titers of anti-CarP antibodies of patients and controls are shown in Figure 2.

Anti-CarP antibodies levels were significantly higher in patients with deformity and erosions in comparison to those without any changes ( $p<0.05)$ (Fig. 3).

\section{Anti-CarP antibodies and DAS 28}

There was a weak but positive correlation between anti-CarP antibodies and DAS 28, which was estimated by Pearson's correlation coefficient $(r)=0.1471 ; p>0.05$.

\section{Anti-CarP antibodies and DMARDs}

There were 18 patients on methotrexate (MTX) alone and 35 on combination of DMARDs with or without methotrexate (methotrexate + sulfasalazine, $n=4$; 
methotrexate + hydroxychloroquine, $n=13$; methotrexate + sulfasalazine + hydroxychloroquine, $n=9$; methotrexate + sulfasalazine + hydroxychloroquine + leflunomide, $n=1$; methotrexate + hydroxychloroquine + leflunomide, $n=2$; methotrexate + sulfasalazine + rituximab, $n=1$; methotrexate + sulfasalazine + hydroxychloroquine + etanercept, $n=1$; sulfasalazine + hydroxychloroquine, $n=1$; sulfasalazine + hydroxychloroquine + leflunomide, $n=1$; hydroxychloroquine + rituximab, $n=1$; hydroxychloroquine + etanercept, $n=1$ ).

There was no statistically significant difference in the serum level of anti-CarP antibodies between the monotherapy group (MTX) and the combination therapy group. We also compared the anti-CarP antibodies levels in patients taking conventional DMARDs and patients taking biological DMARDs. Out of 53 patients, 4 patients were on biological DMARDs (rituximab and etanercept). On comparison, there was no significant difference between these two groups. In our study, there were nine newly diagnosed cases of RA; we estimated anti-CarP antibody levels before starting the treatment with DMARDs and 12 weeks after the introduction of treatment with DMARDs in these patients. Although antiCarP antibody levels after the treatment with DMARDs were lower in comparison to pre-treatment levels, it was not found to be statistically significant.

\section{Anti-CarP antibodies and rheumatoid factor}

There were 33 RF positive patients (RF+) and 20 RF negative patients (RF-) in this study. We compared the presence or absence of rheumatoid factor with the antiCarP levels in the studied group of RA patients. There was no statistically significant differences in anti-Car-P antibody titers between RF- $(236.58 \pm 164.82)$ and RF+ (346.95 \pm 421.41$)$ patients.

\section{Quality of life}

Quality of life of the RA patients was measured by the SF-36 questionnaire. Quality of life was assessed by eight domains: physical function, role limitation due to physical health, role limitation due to emotional health, energy/fatigue, emotional well-being, social functioning, pain, and general health.

\section{Anti-CarP antibodies and quality of life}

There was a weak and not statistically significant negative correlation between all domains of quality of life and serum level of anti-CarP antibodies $(r=-0.02$ to -0.16 There was found a negative correlation between reduction of the levels of anti-CarP antibodies and domains of quality of life score. It is presented in Table II.

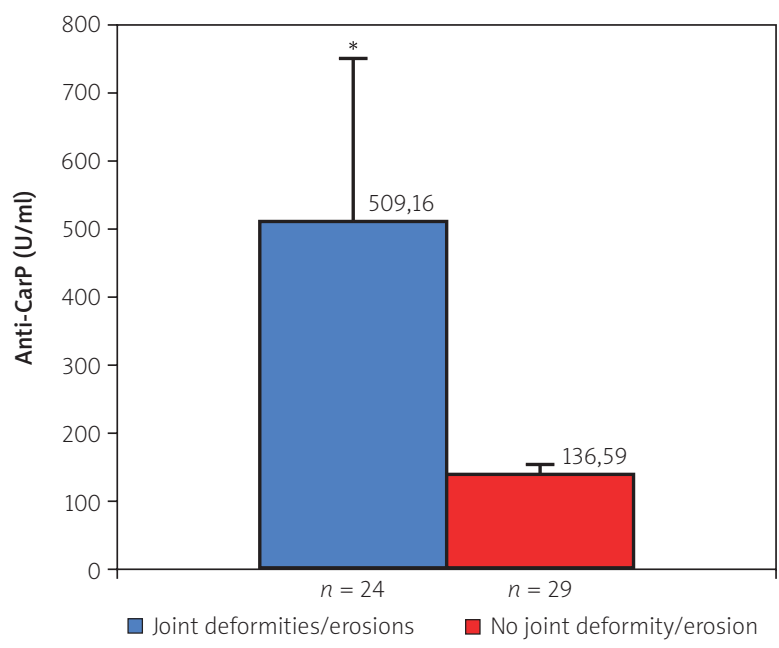

Fig. 3. Comparison of anti-CarP antibody titres in patients of rheumatoid arthritis with and without joint damage. Values are mean $\pm \mathrm{SD}$, compared using two tailed unpaired $t$ test; ${ }^{*} p<0.05$.

\section{Discussion}

The primary aim of this study was to demonstrate a correlation between anti-CarP antibodies and the severity/complications of RA and to study the impact of anti-CarP antibodies on the quality of life in RA patients.

In the current study 22 out of 53 (41.5\%) RA patients had a high titer of anti-CarP antibodies above the cut-off level and were considered positive for anti-CarP antibodies. A similar observation was made in a previous study conducted by Shi et al. in which $43 \%$ of patients with RA were positive for anti-CarP antibodies [7]. We also observed a significant relationship between anti-CarP antibodies and deformity and erosion of bones in the RA patients, but in contrast to ACPA antibodies, which have

Table II. Correlation between anti-CarP antibody titre with different domains of quality of life

\begin{tabular}{lc}
\hline Quality of life domains & $\begin{array}{c}\text { Anti-CarP correlation } \\
\text { coefficient }(r)\end{array}$ \\
\hline Physical function & -0.0786 \\
\hline $\begin{array}{l}\text { Role limitation due to physical } \\
\text { health }\end{array}$ & -0.1141 \\
\hline Role limitation due to emotional & -0.0199 \\
health & -0.1558 \\
\hline Energy/fatigue & -0.1494 \\
\hline Emotional well being & -0.1014 \\
\hline Social functioning & -0.1025 \\
\hline Pain & -0.1428 \\
\hline General health &
\end{tabular}


been observed with respect to direct activation of osteoclasts, there are no such unambiguous results for antiCarP antibodies. This is in accordance with a previous study by Shi et al. which showed that anti-CarP antibodies have a significant association with bone erosion [7] A higher serum level of anti-CarP antibodies may indicate ongoing joint damage. However, in the current study no correlation between serum anti-CarP antibody levels and DAS-28 scores was observed.

This is in accordance with a previous study conducted by Yee et al. [12], in which the authors concluded that there was no individual marker correlating with DAS 28. However, in an earlier study anti-CarP antibodies combined with presence of ACPA fine specificities correlated with DAS 28 [12]. Following treatment with DMARDs there was a reduction of the titers of anti-CarP antibodies, which could have clinical/prognostic significance, but it did not reach statistical significance. Similarly, no statistically significant difference in the level of antiCarP antibodies in RA patients taking methotrexate alone compared with other DMARDs was observed. Furthermore, there was no statistically significant difference in the serum level of anti-CarP antibodies in RA patients taking conventional DMARDs compared with biological DMARDs. As mentioned above, higher antiCarP antibody titers were observed in RF+ patients in comparison to RF- patients, but statistical significance was not found. Present knowledge is unable to explain this weak association.

We assessed the quality of life in RA patients by Short Form questionnaire 36 (SF-36), which is a widely used generic measure of health status. In earlier studies on patients with RA, the SF-36 scales were reliable, correlated with core disease activity measures and were responsive to improvements in health [13]. In the current study SF-36 was administered by an interviewer. There are 8 domains by which we assessed the physical health and mental health of the patients. A significant improvement was observed in all domains of quality of life questionnaire scores following the treatment with DMARDs in patients newly diagnosed with RA. This is in accordance with a previous study by da Mota et al. [14] that showed a significant improvement in Health Assessment Questionnaire ( $\mathrm{HAQ}$ ) and domains of SF-36 after the treatment with DMARDs in RA. Another study conducted by West and Jonsson [15] showed similar results regarding improvement in domains of quality of life in RA. In this study, on comparing the quality of life according to SF-36 in patients who had taken methotrexate alone with those who had taken a combination of methotrexate with other DMARDs, there was no significant difference in the scores. Currently there is a lack of literature describ- ing the correlation between anti-CarP antibodies and quality of life in RA patients; however, this study shows a weak negative correlation between level of anti-CarP antibodies and all domains of quality of life, but it is not statistically significant. Some limitations of the present study have to be pointed out: Firstly, cross-sectional data are presented; and therefore, temporal association between DMARDs and anti-CarP antibodies cannot be established; secondly, the sample size is relatively small and heterogeneous, and thus does not allow comparison between the subgroups (patients treated with biologics versus conventional DMARDs); thirdly, the disease may be responsible for the findings not only via biological mechanisms but also via confounding by other disease-related traits.

\section{Conclusions}

Anti-CarP antibodies levels in RA patients with erosions/deformities were higher in comparison to those without any erosion/deformity, which indicates that anti-CarP antibodies may have prognostic value in predicting joint damage. There is no correlation between disease activity and anti-CarP antibody levels in patients with RA. Quality of life in RA patients following 12 weeks of treatment with DMARDs improved, but it had no correlation with the serum levels of anti-CarP antibodies. Considering the above, it appears that antiCarP antibodies primarily may be a useful biomarker for estimating the risk of erosions and thus progression of radiological changes in the course of RA.

\section{Acknowledgement}

The authors are thankful to Dr. D.K. Nim for help in data analysis.

The authors declare no conflict of interest.

This study is registered with Clinical Trials Registry, India, No. CTRI/2015/06/005878.

\section{References}

1. Rheumatoid Arthritis - Fast Facts [Internet]. American college of rheumatology, 2015 [cited 2015]. Available from: http:// www.rheumatology.org/I-Am-A/Patient-Caregiver/Diseases-Conditions/Rheumatoid-Arthritis.

2. Chronic diseases and health promotion- Chronic rheumatic conditions Geneva, Switzerland: World Health Organization 2015. Available from: http://www.who.int/chp/topics/rheumatic/en/.

3. Raza K, Falciani F, Curnow SJ, et al. Early rheumatoid arthritis is characterized by a distinct and transient synovial fluid cytokine profile of T cell and stromal cell origin. Arthritis Res Ther 2005; 7: R784-795. 
4. Singh JA, Furst DE, Bharat A, et al. 2012 update of the 2008 American College of Rheumatology recommendations for the use of disease-modifying antirheumatic drugs and biologic agents in the treatment of rheumatoid arthritis. Arthritis Care Res (Hoboken) 2012; 64: 625-639.

5. Nienhuis RL, Mandema E. A New Serum Factor in Patients with Rheumatoid Arthritis; the Antiperinuclear Factor. Ann Rheum Dis 1964; 23: 302-305.

6. Rantapaa-Dahlqvist S, de Jong BA, Berglin E, et al. Antibodies against cyclic citrullinated peptide and IgA rheumatoid factor predict the development of rheumatoid arthritis. Arthritis Rheum 2003; 48: 2741-2749.

7. Shi J, Knevel R, Suwannalai P, et al. Autoantibodies recognizing carbamylated proteins are present in sera of patients with rheumatoid arthritis and predict joint damage. Proc Natl Acad Sci U S A 2011; 108: 17372-17377.

8. Girbal-Neuhauser E, Durieux JJ, Arnaud M, et al. The epitopes targeted by the rheumatoid arthritis-associated antifilaggrin autoantibodies are posttranslationally generated on various sites of (pro)filaggrin by deimination of arginine residues. J Immunol 1999; 162: 585-594.

9. Schellekens GA, de Jong BA, van den Hoogen FH, et al. Citrulline is an essential constituent of antigenic determinants rec- ognized by rheumatoid arthritis-specific autoantibodies. J Clin Invest 1998; 101: 273-281.

10. Ware JE Jr., Gandek B. Overview of the SF-36 Health Survey and the International Quality of Life Assessment (IQOLA) Project. J Clin Epidemiol 1998; 51: 903-912.

11. Prevoo ML, van't Hof MA, Kuper $\mathrm{HH}$, et al. Modified disease activity scores that include twenty-eight-joint counts. Development and validation in a prospective longitudinal study of patients with rheumatoid arthritis. Arthritis Rheum 1995; 38: 44-48.

12. Yee A, Webb T, Seaman A, et al. Anti-CarP antibodies as promising marker to measure joint damage and disease activity in patients with rheumatoid arthritis. Immunol Res 2015; 61: 24-30.

13. Pollard L, Choy EH, Scott DL. The consequences of rheumatoid arthritis: quality of life measures in the individual patient. Clin Exp Rheumatol 2005; 23: S43-52.

14. Mota LMH, Laurindo IMM, Santos Neto LL. Avaliação prospectiva da qualidade de vida em uma coorte de pacientes com artrite reumatoide inicial. Rev Bras Reumatol 2010; 50: 249-261.

15. West E, Jonsson SW. Health-related quality of life in rheumatoid arthritis in Northern Sweden: a comparison between patients with early RA, patients with medium-term disease and controls, using SF-36. Clin Rheumatol 2005; 24: 117-122. 